The height of watermelons with wall Extended Abstract 


\title{
A new method for computing asymptotics of diagonal coefficients of multivariate generating functions
}

\author{
Alexander Raichev and Mark C. Wilson \\ Department of Computer Science \\ University of Auckland \\ Private Bag 92019 \\ Auckland \\ New Zealand \\ received $19 \mathrm{Feb} 2007$, revised $19^{\text {th }}$ January 2008 ,
}

Let $\sum_{\mathbf{n} \in \mathbb{N}^{d}} F_{\mathbf{n}} \mathbf{x}^{\mathbf{n}}$ be a multivariate generating function that converges in a neighborhood of the origin of $\mathbb{C}^{d}$. We present a new, multivariate method for computing the asymptotics of the diagonal coefficients $F_{a_{1} n, \ldots, a_{d} n}$ and show its superiority over the standard, univariate diagonal method. Several examples are given in detail.

Keywords: generating function, multivariate, asymptotics, diagonal

\section{Introduction}

Before beginning, let us set some notation. Boldface letters denote row vectors, with the symbols $\mathbf{0}$ and 1 denoting the vectors of all zeros and all ones, respectively. Component $i$ of a vector $\mathbf{x}$ is denoted by $x_{i}$. We also use multi-index notation, so that $\mathbf{x}^{\mathbf{n}}=x_{1}^{n_{1}} \cdots x_{d}^{n_{d}}$. Lastly, $\partial_{i}:=\partial / \partial x_{i}$.

Let $F(\mathbf{x})=\sum_{\mathbf{n} \in \mathbb{N}^{d}} F_{\mathbf{n}} \mathbf{x}^{\mathbf{n}}$ be a complex power series that converges in a neighborhood of the origin but not on all of $\mathbb{C}^{d}$. We wish to compute asymptotics for the diagonal coefficients $F_{a_{1} n, \ldots, a_{d} n}$ for fixed positive integers $a_{1}, \ldots, a_{d}$, a task often useful in enumerative combinatorics. For simplicity of presentation, we suppose that $F_{\mathbf{n}} \geq 0$ and that $F$ is rational, although much greater generality is possible. Thus far the only general method to extract diagonal asymptotics to be found in the literature is what we call the standard diagonal method. It consists of two steps: (1) find a closed form or defining equation for the diagonal generating function $G(x):=\sum_{n \in \mathbb{N}} F_{a_{1} n, \ldots, a_{d} n} x^{n}$ via contour integration (in the style of (Fur67) and (HK71) and summarized in (Sta99, Section 6.3)); (2) apply univariate singularity analysis to $G(x)$ (in the style of (FS) for instance) to compute the asymptotics. However, as elaborated below, this method is quite limited, working well only for main diagonals in two variables, that is, for computing the asymptotics of $F_{n n}$.

In this article we present a new, more versatile diagonal method. It consists of one step: apply multivariate singularity analysis (in the style of ( $(\overline{\mathrm{PW} 02}),(\overline{\mathrm{PW} 04}),(\overline{\mathrm{BP}})$ ) directly to $F(\mathbf{x})$ to compute the 
asymptotics. In some sense, the results of the present paper are implicit in the above papers. However in our opinion, the message that the standard diagonal method is not workable in all but the simplest problems, and the method presented here is clearly preferable to it, has not yet penetrated far into the Analysis of Algorithms community.

\section{Limitations of the Standard Diagonal Method}

What is wrong with the standard diagonal method? It certainly works well for computing main diagonal asymptotics in two variables, as witnessed by the following example taken from (MZS04).

Example 2.1 (Zigzag-free Binary Words) The bivariate generating function

$$
F(x, y)=\sum_{m, n} F_{m n} x^{m} y^{n}=\frac{1+x y+x^{2} y^{2}}{1-x-y+x y-x^{2} y^{2}}
$$

counts the number of words over a binary alphabet, $\{0,1\}$ say, that have $m$ zeros and $n$ ones and do not contain zigzags, that is, the subwords 010 and 101. The main diagonal coefficients $F_{n n}$, then, count zigzag-free binary words with an equal number of zeros and ones.

To compute the asymptotics of $F_{n n}$ using the standard diagonal method we proceed as follows. Since $F(x, y)$ is rational and holomorphic in a neighborhood of the origin, for fixed $x$ small enough $F(x / t, t)$ will be rational and holomorphic as a function of $t$ in some annulus about $t=0$. Thus in that annulus it can be represented by a Laurent series whose constant term is $\left[t^{0}\right] F(x / t, t)=\sum_{n \geq 0} F_{n n} x^{n}$, the series we want. By Cauchy's integral formula and the residue theorem we have that for some circle $\gamma_{x}$ about $t=0$

$$
\begin{aligned}
\sum_{n} F_{n n} x^{n} & =\left[t^{0}\right] F(x / t, t) \\
& =\frac{1}{2 \pi \mathrm{i}} \int_{\gamma_{x}} \frac{F(x / t, t)}{t} \mathrm{~d} t \\
& =\sum_{k} \operatorname{Res}\left(F(x / t, t) / t ; t=s_{k}\right),
\end{aligned}
$$

where the $s_{k}$ are the "small" singularities of $F(x / t, t) / t$, that is, the ones satisfying $\lim _{x \rightarrow 0} s_{k}(x)=0$. Since $F$ is rational, these singularities are poles and algebraic functions of $x$, so that the residue sum, the diagonal generating function, is also an algebraic function of $x$.

In particular,

$$
F(x / t, t) / t=\frac{1+x+x^{2}}{-t^{2}+\left(1+x-x^{2}\right) t-x},
$$

which has a single simple pole approaching zero as $x$ approaches 0 , namely, $s=\frac{1}{2}\left(1+x-x^{2}-\right.$ $\left.\sqrt{1-2 x-x^{2}-2 x^{3}+x^{4}}\right)$. The residue, the diagonal generating function, is then

$$
G(x):=\sqrt{\frac{x^{2}+x+1}{x^{2}-3 x+1}} .
$$


Now, the singularity of $G$ closest to the origin is $\omega:=(3-\sqrt{5}) / 2$, and its reciprocal is the exponential growth order of the coefficients of $G$. To determine the leading subexponential factor we note that

$$
G(x) \sim \frac{1}{\sqrt{1-\frac{x}{\omega}}}\left(\sqrt{\frac{x^{2}+x+1}{-\omega\left(x-\frac{3+\sqrt{5}}{2}\right)}}\right)_{x=\omega}=\left(1-\frac{x}{\omega}\right)^{-1 / 2} \frac{2}{\sqrt{\sqrt{5}}}
$$

as $x \rightarrow \omega$, so that

$$
F_{n n}=G_{n} \sim \omega^{-n} \frac{2}{\sqrt{\sqrt{5}}} \frac{n^{1 / 2-1}}{\Gamma(1 / 2)}=\left(\frac{2}{3-\sqrt{5}}\right)^{n} \frac{2}{\sqrt{\sqrt{5} \pi n}}
$$

by asymptotic transfer (see (FS, Chapter VI) for instance).

However, the standard diagonal method encounters major problems off the main diagonal even in two variables, as illustrated in the next example adapted from (Sta99, Section 6.3).

Example 2.2 (Lattice Paths) The bivariate generating function

$$
F(x, y)=\frac{1}{1-x-y-x y},
$$

whose coefficients $F_{m n}$ are called the Delannoy numbers, counts the number of lattice paths from $(0,0)$ to $(m, n)$ with allowable steps $(1,0),(0,1)$, and $(1,1)$.

To compute the asymptotics of the general diagonal coefficients $F_{a n, b n}$ using the standard diagonal method, we fix $x>0$ small enough and try to find the small poles of

$$
\begin{aligned}
G(x) & :=\sum_{n} F_{a n, b n} x^{n} \\
& =\left[t^{0}\right] F\left(x^{1 / a} / t^{b}, t^{a}\right) \\
& =\frac{1}{2 \pi \mathrm{i}} \int_{\gamma_{x}} \frac{F\left(x^{1 / a} / t^{b}, t^{a}\right)}{t} \mathrm{~d} t \\
& =\frac{1}{2 \pi \mathrm{i}} \int_{\gamma_{x}} \frac{t^{b-1}}{t^{b}-x^{1 / a}-t^{a+b}-x^{1 / a} t^{a}} \mathrm{~d} t
\end{aligned}
$$

Since $t^{b}-x^{1 / a}-t^{a+b}-x^{1 / a} t^{a}$ has a zero of multiplicity $b$ at $t=0$ when $x=0$, it follows that $F\left(x^{1 / a} / t^{b}, t^{a}\right) / t$ has a single pole $s$ of order $b$ satisfying $\lim _{x \rightarrow 0} s(x)=0$. Thus we have that

$$
\begin{aligned}
G(x) & =\operatorname{Res}\left(F\left(x^{1 / a} / t^{b}, t^{a}\right) / t ; t=s\right) \\
& =\lim _{t \rightarrow s} \frac{1}{(b-1) !} \partial_{t}^{b-1}\left((t-s)^{b} F\left(x^{1 / a} / t^{b}, t^{a}\right) / t\right),
\end{aligned}
$$

where $\partial_{t}$ is the derivative with respect to $t$. Patiently tracing through Leibniz's rule for the iterated derivative of a product, we could express this limit in terms of $s$ and $g(t):=(t-s)^{-b}\left(t^{b}-x^{1 / a}-t^{a+b}\right.$ $\left.x^{1 / a} t^{a}\right)$ and use it to find an algebraic equation satisfied by $G$. However, even with the help of Maple, this seems unlikely for general $a$ and $b$.

Thus, already at the first step of the standard diagonal method, it seems we are thwarted, the cause of the problem being the parameters $a$ and b occurring in the exponent: the larger they get, the greater the difficulty in finding a defining equation for $G$. 
So we should keep to main diagonal asymptotics when using the standard diagonal method. But even this poses problems in three or more variables, as illustrated by the next example.

Example 2.3 (Ternary Words) The trivariate generating function

$$
F(x, y, z)=\frac{1}{1-x-y-z}
$$

counts the the number of words $F_{l m n}$ over a ternary alphabet, $\{0,1,2\}$ say, that have $l$ zeros, $m$ ones, and $n$ twos (an easy consequence of the symbolic method as described in (FS)). A simple combinatorial argument shows that $F_{l m n}=\left(\begin{array}{c}l+m+n \\ l, m, n\end{array}\right)$, to which one could apply Stirling's formula to derive the asymptotics.

To compute the asymptotics of the main diagonal coefficients $F_{n n n}$ using the standard diagonal method instead, we iterate the contour integration process. First

$$
\begin{aligned}
\sum_{n} F_{m, n, n} x^{m} y^{n} & =\left[t^{0}\right] F(x, y / t, t) \\
& =\frac{1}{2 \pi \mathrm{i}} \int_{\gamma_{x}} \frac{F(x, y / t, t)}{t} \mathrm{~d} t \\
& =\frac{1}{2 \pi \mathrm{i}} \int_{\gamma_{x}} \frac{1}{-t^{2}+(1-x) t-y} \mathrm{~d} t
\end{aligned}
$$

which has a single simple pole approaching zero as $y$ and $x$ approach 0 , namely, $s=\frac{1}{2}(1+x-$ $\left.\sqrt{1-2 x+x^{2}-4 y}\right)$. The residue is then

$$
G(x, y):=\frac{1}{\sqrt{1-2 x+x^{2}-4 y}} .
$$

Second, since

$$
\lim _{x \rightarrow 0} G(x / t, t)=\lim _{x \rightarrow 0} \frac{1}{\sqrt{1-2 x / t+x^{2} / t^{2}-4 t}}=\frac{1}{\sqrt{1-4 t}},
$$

$G(x / t, t)$ is a holomorphic function of $t$ in some annulus about $t=0$. Thus computing

$$
\frac{1}{2 \pi \mathrm{i}} \int_{\gamma_{x}} \frac{G(x / t, t)}{t} \mathrm{~d} t=\frac{1}{2 \pi \mathrm{i}} \int_{\gamma_{x}} \frac{1}{t \sqrt{1-2 x / t+x^{2} / t^{2}-4 t}} \mathrm{~d} t
$$

will give us the diagonal generating function. Proceeding, we employ Maple and find that two singularities of $G(x / t, t) / t$ approach 0 as $x$ approaches 0 , namely

$$
-\frac{1}{24} p(x)+\frac{q(x)}{p(x)}+\frac{1}{12}+\frac{1}{2} \mathrm{i} \sqrt{3}\left(\frac{1}{12} p(x)+\frac{q(x)}{p(x)}\right)
$$

and its conjugate, where $p(x)=\left(-36 x+216 x^{2}+1+24 \sqrt{-3 x^{3}+81 x^{4}}\right)^{1 / 3}$ and $q(x)=x-\frac{1}{24}$. While both of these singularities are algebraic in $x$, Maple does not compute the sum of their residues in reasonable time. 
The problem is that the residue sum, that is, the diagonal generating function $H(x)$, say, is not algebraic: as mentioned above, by means other than the diagonal method we know that $H(x)=\sum\left(\begin{array}{c}3 n \\ n, n, n\end{array}\right) x^{n}$, which is not algebraic (see (Sta99. Exercise solution 6.3) for instance). While $H$ is D-finite - the diagonal of any rational function in any finite number of variables is D-finite (Lip88) - it is unclear how to find its closed form or defining differential equation. (With the extra knowledge that $H(x)=\sum\left(\begin{array}{c}3 n \\ n, n, n\end{array}\right) x^{n}$ and using the Maple package Mgfun, we get that $27 x(x-1) H^{\prime \prime}(x)+(54 x-1) H^{\prime}(x)+6 H(x)=0$, which has a hypergeometric solution). Moreover, even given the defining differential equation, we still require the theory of univariate singularity analysis of D-finite functions to compute the asymptotics. But this theory has not been worked out in general. Indeed, as Philippe Flajolet has informed us, certain aspects of it, such as the so-called connection problem, might not be computable!

\section{A New Diagonal Method}

To transcend the limitations of the standard diagonal method, to go beyond main diagonal asymptotics of bivariate generating functions, we take a multivariate approach. When the diagonal method works, it does at least produce an explicit algebraic formula for the diagonal generating function, which our method here does not. However, it is normally much more useful to have an asymptotic expression for the coefficients. To this end, we apply a multivariate singularity analysis directly to $F(\mathbf{x})$. Such an analysis has been recently developed by Baryshnikov, Pemantle, and Wilson in (PW02), (PW04), and (BP), the relevant parts of which we now summarize and adapt to our needs ${ }^{(i)}$

Let $\mathcal{D} \subset \mathbb{C}^{d}$ be the open domain of convergence of $F$ and write $F(\mathbf{x})=I(\mathbf{x}) / J(\mathbf{x})$ for some $I$ and $J$ holomorphic on an open domain $\mathcal{D}^{\prime}$ containing the closure of $\mathcal{D}$, and relatively prime in the ring of holomorphic functions on $\mathcal{D}^{\prime}$. In all examples in this paper, the representation of $F$ as a quotient in fact holds on all of $\mathbb{C}^{d}$, but the extra generality is sometimes useful in applications. Let $\mathcal{V}$ be the complex variety $\left\{\mathbf{x} \in \mathbb{C}^{d}: J(\mathbf{x})=0\right\}$.

A critical point of $F$ for $\mathbf{n} \in\left(\mathbb{N}^{+}\right)^{d}$ is a solution of

$$
\begin{aligned}
J(\mathbf{x}) & =0 \\
n_{d} x_{i} \partial_{i} J(\mathbf{x}) & =n_{i} x_{d} \partial_{d} J(\mathbf{x}) \quad(i<d) .
\end{aligned}
$$

Let CRIT(n) denote the set of all critical points of $F$ for $\mathbf{n}$. For generic directions $\mathbf{n}$, this set is finite, being a zero-dimensional complex variety. The main situation in which CRIT(n) is infinite occurs when $J$ defines a binomial variety $\left\{\mathbf{x} \mid \mathbf{x}^{\mathbf{a}}-\mathbf{x}^{\mathbf{b}}\right\}$, in which case CRIT(n) is empty for all but one direction and uncountable otherwise. Such examples can be analysed by a variant of the methods shown here.

A contributing point of $F$ for $\mathbf{n}$ is a critical point that influences the asymptotics of the coefficients of $F$ in the direction of $\mathbf{n}$. Let $\operatorname{Contrib}(\mathbf{n})$ denote the set of all such points. While $\operatorname{ContRiB}(\mathbf{n})$ is ill-defined here, its functional role will become clear from the next two theorems.

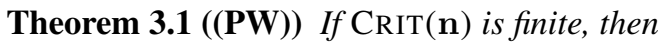

- $\operatorname{CRIT(n)~contains~exactly~one~point,~call~it~} \mathbf{c}$, that lies in the positive orthant of $\mathbb{R}^{d}$, and $\mathbf{c} \in$ $\operatorname{CONTRIB}(\mathbf{n})$;

(i) As a whole, the multivariate singularity analysis developed in the aforementioned articles is much more general than what we present in this article, as it applies not only to rational functions but also to locally meromorphic functions. For a more complete summary including further examples, see the forthcoming article (PW). 
- all other members of CONTRIB(n) lie on $T(\mathbf{c})$;

- all members $\mathbf{p}$ of $\operatorname{CONTRIB}(\mathbf{n})$ satisfy $\mathcal{V} \cap D(\mathbf{p}) \subseteq T(\mathbf{p})$;

- in the case where $J=1-P$ for some aperiodic power series with nonnegative coefficients $P$, $\operatorname{CONTRIB}(\mathbf{n})=\{\mathbf{c}\}$.

Here $T(\mathbf{p})$ and $D(\mathbf{p})$ denote respectively the torus and the polydisk of a point $\mathbf{p} \in \mathbb{C}^{d}$, that is, respectively the sets $\left\{\mathbf{x} \in \mathbb{C}^{d}: \forall i \leq d\left|x_{i}\right|=\left|p_{i}\right|\right\}$ and $\left\{\mathbf{x} \in \mathbb{C}^{d}: \forall i \leq d\left|x_{i}\right| \leq\left|p_{i}\right|\right\}$, and a power series is aperiodic if the $\mathbb{Z}$-span of its monomial vectors is all of $\mathbb{Z}^{d}$.

A point $\mathbf{c} \in \mathcal{V}$ is a smooth point if $\mathcal{V}$ is a smooth complex manifold in a neighborhood of $\mathbf{c}$, or equivalently, if $\partial_{i} J(\mathbf{c}) \neq 0$ for some $i$ (see (BK86, page 363) for instance). For simplicity of presentation, we deal only with smooth points in this article. This is the generic case, although interesting examples are not always generic. For more on the case of non-smooth points, see (PW04).

Theorem 3.2 $((\overline{\mathbf{P W}}))$ Let $\mathbf{n}=\left(a_{1} n, \ldots, a_{d} n\right)$ for some $a_{1}, \ldots, a_{d} \in \mathbb{N}^{+}$. If $\operatorname{CONTRIB}(\mathbf{n})$ consists of a single smooth point $\mathbf{c}$ such that $c_{d} \partial_{d} J(\mathbf{c}) \neq 0$, say, and $c_{d}$ is a simple zero of $x_{d} \mapsto J\left(c_{1}, \ldots, c_{d-1}, x_{d}\right)$, then

$$
F_{\mathbf{n}}=\mathbf{c}^{-\mathbf{n}}\left(\sum_{j=0}^{j^{\prime}} b_{j} n_{d}^{(1-d-j) / 2}+O\left(n_{d}^{-d-j^{\prime}}\right)\right)
$$

for some $b_{j} \in \mathbb{C}$ as $n \rightarrow \infty$. If $\operatorname{CONTRIB}(\mathbf{n})$ consists of a finite set of smooth points $\mathbf{c}_{1}, \ldots, \mathbf{c}_{r}$ each satisfying the hypotheses above, then

$$
F_{\mathbf{n}}=\sum_{i} \mathbf{c}_{i}^{-\mathbf{n}}\left(\sum_{j=0}^{j^{\prime}} b_{i j} n_{d}^{(1-d-j) / 2}+O\left(n_{d}^{-d-j^{\prime}}\right)\right)
$$

as $n \rightarrow \infty$.

Theorem 3.3 In Theorem 3.2

$$
b_{0}=\frac{I(\mathbf{c})}{-c_{d} \partial_{d} J(\mathbf{c}) \sqrt{(2 \pi)^{d-1} \operatorname{det} \mathbf{H}}},
$$

assuming $\operatorname{det} \mathbf{H} \neq 0$. Here $\mathbf{H}$ is the matrix whose entries are

$$
\begin{aligned}
\mathbf{H}_{l m} & =\left.\frac{c_{l} c_{m}}{c_{d}^{2}\left(\partial_{d} J\right)^{2}}\left(\partial_{m} J \partial_{l} J+c_{d}\left(\partial_{d} J \partial_{m} \partial_{l} J-\partial_{m} J \partial_{d} \partial_{l} J-\partial_{l} J \partial_{m} \partial_{d} J+\frac{\partial_{l} J \partial_{m} J}{\partial_{d} J} \partial_{d}^{2} J\right)\right)\right|_{\mathbf{x}=\mathbf{c}} ; \\
\mathbf{H}_{l l} & =\frac{c_{l} \partial_{l} J}{c_{d} \partial_{d} J}+\left.\frac{c_{l}^{2}}{c_{d}^{2}\left(\partial_{d} J\right)^{2}}\left(\left(\partial_{l} J\right)^{2}+c_{d}\left(\partial_{d} J \partial_{l}^{2} J-2 \partial_{l} J \partial_{d} \partial_{l} J+\frac{\left(\partial_{l} J\right)^{2}}{\partial_{d} J} \partial_{d}^{2} J\right)\right)\right|_{\mathbf{x}=\mathbf{c}}
\end{aligned}
$$

where $l, m<d$ and $l \neq m$.

Proof: The formula for $b_{0}$ comes from (PW02, Theorem 3.5). (Please note the typo therein: the $z_{d} H_{d}$ should be $\mathrm{a}-z_{d} H_{d}$.) We prove the formula for $\mathbf{H}$ only. 
For easy reading, let $\widehat{\mathbf{x}}=\left(x_{1}, \ldots, x_{d-1}\right)$. Since $J$ is holomorphic and $\partial_{d} J(\mathbf{c}) \neq 0$, there exists a holomorphic function $g$ guaranteed by the implicit function theorem such that in some open ball around $\mathbf{c}, J(\widehat{\mathbf{x}}, g(\widehat{\mathbf{x}}))=0$. As shown in (PW02), $\mathbf{H}$ is the Hessian (the matrix of second partial derivatives) of

$$
\widetilde{h}(\widehat{\boldsymbol{\theta}}):=\log g\left(c_{1} \mathrm{e}^{\mathrm{i} \theta_{1}}, \ldots, c_{d-1} \mathrm{e}^{\mathrm{i} \theta_{d-1}}\right)+\mathrm{i} \sum_{j=1}^{d-1} \frac{n_{j}}{n_{d}} \theta_{j}-\log g(\mathbf{c})
$$

evaluated at $\widehat{\boldsymbol{\theta}}=\mathbf{0}$.

Now, from the implicit function theorem we also get

$$
\begin{aligned}
\partial_{l} g(\widehat{\mathbf{x}}) & =-\frac{\partial_{l} J(\widehat{\mathbf{x}}, g(\widehat{\mathbf{x}}))}{\partial_{d} J(\widehat{\mathbf{x}}, g(\widehat{\mathbf{x}}))} \quad(l<d) \\
\partial_{m} \partial_{l} J(\widehat{\mathbf{x}}, g(\widehat{\mathbf{x}})) & =\partial_{m} \partial_{l} J+\partial_{d} \partial_{l} J \partial_{m} g=\partial_{m} \partial_{l} J-\partial_{d} \partial_{l} J \frac{\partial_{m} J}{\partial_{d} J} \quad(l \leq d, m<d) .
\end{aligned}
$$

Thus, up to an additive constant, $\partial_{l} \widetilde{h}=-\mathrm{i} c_{l} \mathrm{e}^{i \theta_{l}} g^{-1} \partial_{l} J / \partial_{d} J$, and for $l, m<d$ with $l \neq m$,

$$
\begin{aligned}
\left.\partial_{m} \partial_{l} \tilde{h}\right|_{\widehat{\boldsymbol{\theta}}=\mathbf{0}} & =\left.c_{l} c_{m}\left(-g^{-2} \partial_{m} g \frac{\partial_{l} J}{\partial_{d} J}+g^{-1} \frac{\partial_{d} J \partial_{m} \partial_{l} J-\partial_{l} J \partial_{m} \partial_{d} J}{\left(\partial_{d} J\right)^{2}}\right)\right|_{\mathbf{x}=\mathbf{c}} ; \\
\left.\partial_{l}^{2} \widetilde{h}\right|_{\widehat{\boldsymbol{\theta}}=\mathbf{0}} & =c_{l} g^{-1} \frac{\partial_{l} J}{\partial_{d} J}+\left.c_{l}^{2}\left(-g^{-2} \partial_{l} g \frac{\partial_{l} J}{\partial_{d} J}+g^{-1} \frac{\partial_{d} J \partial_{l} \partial_{l} J-\partial_{l} J \partial_{l} \partial_{d} J}{\left(\partial_{d} J\right)^{2}}\right)\right|_{\mathbf{x}=\mathbf{c}}
\end{aligned}
$$

The result then follows by plugging in the implicit function theorem equations above and simplifying.

Several of our examples involve main diagonal asymptotics for symmetric $J$. In this case the matrix $\mathbf{H}$ simplifies greatly.

Proposition 3.4 If $\operatorname{CRIT}(\mathbf{n})$ is finite, $\mathbf{n}=(n, \ldots, n), J(\mathbf{x})$ is symmetric in $\mathbf{x}$, and $\partial_{d} J(\mathbf{c}) \neq 0$, where $\mathbf{c}$ is the contributing point that lies in the positive orthant of $\mathbb{R}^{d}$, then $\mathbf{c}=(c, \ldots, c)$ for some positive real $c$, and

$$
\begin{aligned}
\mathbf{H}_{l m} & =a, \\
\mathbf{H}_{l l} & =2 a, \quad \text { and } \\
\operatorname{det} \mathbf{H} & =d a^{d-1},
\end{aligned}
$$

where $a=1+\left.\frac{c}{\partial_{d} J}\left(\partial_{d}^{2} J-\partial_{1} \partial_{d} J\right)\right|_{\mathbf{x}=\mathbf{c}}, l, m<d$, and $l \neq m$.

Proof: By Theorem 3.1, CRIT(n) contains exactly one point $\mathbf{c}$ that lies in the positive orthant of $\mathbb{R}^{d}$. By the symmetry of the critical equations $J=0$ and $x_{i} \partial_{i} J=x_{d} \partial_{d} J(i<d)$ induced by the symmetry of $\mathbf{n}$ and $J$, any permutation of c's coordinates is also a critical point lying in the positive orthant of $\mathbb{R}^{d}$. Since there can be only one such point, $\mathbf{c}=(c, \ldots, c)$ for some positive real $c$. 
Since $J$ is symmetric and $\mathbf{c}=(c, \ldots, c)$, all first partial derivatives of $J$ are equal at $\mathbf{c}$, all mixed second partial derivatives of $J$ are equal at $\mathbf{c}$, and all diagonal second partial derivatives of $J$ are equal at c. Thus the entries of $\mathbf{H}$ simplify to

$$
\begin{aligned}
\left.\partial_{m} \partial_{l} \widetilde{h}\right|_{\widehat{\boldsymbol{\theta}}=\mathbf{0}} & =1+\left.\frac{c}{\partial_{d} J}\left(\partial_{d}^{2} J-\partial_{1} \partial_{d} J\right)\right|_{\mathbf{x}=\mathbf{c}}=: a \\
\left.\partial_{l}^{2} \widetilde{h}\right|_{\widehat{\boldsymbol{\theta}}=\mathbf{0}} & =2 a
\end{aligned}
$$

where $l, m<d$ and $l \neq m$. Thus the eigenvalues of (the real symmetric, hence diagonalizable matrix) $(1 / a) \mathbf{H}$ are 1 and $d$ with eigenspace dimensions $d-2$ and 1 , respectively, so that $\operatorname{det} \mathbf{H}=a^{d-1} d$.

Our new diagonal method is simply the application of Theorems 3.1, 3.2, and 3.3 directly to $F$. We illustrate this now with several examples.

Example 3.5 (Zigzag-free Binary Words) Consider again the bivariate generating function

$$
F(x, y)=\sum_{m, n} F_{m n} x^{m} y^{n}=\frac{1+x y+x^{2} y^{2}}{1-x-y+x y-x^{2} y^{2}}
$$

of Section 2. To compute the asymptotics of $F_{n n}$ using the new diagonal method, let $\mathbf{n}=(n, n)$ and $J=1-x-y+x y-x^{2} y^{2}$. Then $\operatorname{CRIT}(\mathbf{n})$, the solution set of $J=0$ and $n x \partial_{x} J=n y \partial_{y} J$, comprises $(1 / \phi, 1 / \phi),(-\phi,-\phi),(1 \pm \mathrm{i} \sqrt{3}) / 2$, and $(1 \pm \mathrm{i} \sqrt{3}) / 2$, where $\phi=(1+\sqrt{5}) / 2$. By Theorem 3.1 $\mathbf{c}:=$ $(1 / \phi, 1 / \phi)$ is a contributing point and the only such point, since none of the other critical points lie on the same torus as $\mathbf{c}$. It is also a smooth point. By Proposition 3.4 . $\operatorname{det} \mathbf{H}=4 /(-5+3 \sqrt{5})$, and by Theorems 3.2 and 3.3

$$
F_{n n} \sim \phi^{2 n} \frac{2}{\sqrt{\sqrt{5} \pi n}}
$$

This agrees with our answer in Section 2 since $\phi^{2}=2 /(3-\sqrt{5})$.

Example 3.6 (Lattice Paths) Consider again the bivariate generating function

$$
F(x, y)=\frac{1}{1-x-y-x y}
$$

of Section 2. To compute the asymptotics of $F_{a n, b n}$ using the new diagonal method, let $\mathbf{n}=($ an, bn $)$ and $J=1-x-y-x y$. Then CRIT(n), the solution set of $J=0$ and bnx $\partial_{x} J=$ any $\partial_{y} J$, comprises $\left(\frac{-a \pm L}{b}, \frac{-b \pm L}{a}\right)$, where $L=\sqrt{a^{2}+b^{2}}$. By Theorem 3.1 $\mathbf{c}:=\left(\frac{-a+L}{b}, \frac{-b+L}{a}\right)$ is the only contributing point. It is also a smooth point. By Theorem $3.3 \operatorname{det} \mathbf{H}=\frac{-2(b-L) a\left(a^{2}+b^{2}-a L\right)}{\left.(a-L)^{2}(a-b+L)^{2}\right)}$, and by Theorem 3.2 .

$$
F_{a n, b n} \sim\left(\frac{-a+L}{b}\right)^{-a n}\left(\frac{-b+L}{a}\right)^{-b n} \sqrt{\frac{a b}{L(a+b-L)^{2} 2 \pi n}} .
$$

Here, where the standard diagonal method failed, the new diagonal method allowed us to compute the asymptotics quite easily (with the help of Maple). This success comes from a general attribute of the new diagonal method: the parameters a and b appear as factors instead of as powers in the equations we need to solve. 
Example 3.7 (Ternary Words) Consider again the trivariate generating function

$$
F(x, y, z)=\frac{1}{1-x-y-z}
$$

of Section 2 To compute the asymptotics of $F_{a n, b n, c n}$, let $\mathbf{n}=($ an, bn, cn $)$ and $J=1-x-y-z$. Then CRIT(n), the solution set of $J=0, c n x \partial_{x} J=a n z \partial_{z} J$, and cny $\partial_{y} J=b n z \partial_{z} J$ comprises $\mathbf{c}:=(a+b+c)^{-1}(a, b, c)$. By Theorem 3.1. $\mathbf{c}$ is the only contributing point. It is also a smooth point. By Proposition 3.4 $\operatorname{det} \mathbf{H}=a b(a+b+c) / c^{3}$, and by Theorems 3.2 and 3.3

$$
F_{a n, b n, c n} \sim \frac{(a+b+c)^{(a+b+c) n}}{a^{a n} b^{b n} c^{c n}} \sqrt{\frac{a+b+c}{a b c}} \frac{1}{2 \pi n},
$$

in agreement with Stirling's formula.

Here, where the standard diagonal method failed because of the nonalgebraic character of the diagonal generating function, the new diagonal method gave the answer easily, even off the main diagonal.

Rather than multiply examples unnecessarily, we finish with an example involving an arbitrary number of variables. Many more examples of multivariate asymptotics using similar methods can be found in (PW).

Example 3.8 (Alignments) In a problem from computational biology, one is given a finite set of strings of various lengths over some finite alphabet and needs to count the number of ways of inserting blanks into these strings to make them all of equal length. The strings represent genetic sequences, and inserting blanks represents aligning genetically similar segments among the sequences.

More specifically and abstractly, $a\left(d, r_{1}, \ldots, r_{d}\right)$-alignment is a table of $d\{0,1\}$-strings, one in each row, such that the sum of the entries of row $j$ equals $r_{j}(1 \leq j \leq d)$ and no column contains all zeros. (The ones correspond to letters of the genetic sequence and the zeros to blanks.) As shown in (GHOW90), the generating function for the number of $(d, \cdot)$-alignments is

$$
F\left(x_{1}, \ldots, x_{d}\right)=\frac{1}{2-\prod_{i=1}^{d}\left(1+x_{i}\right)} .
$$

To compute the asymptotics of $F_{n, \ldots, n}$ (the case where all rows have the same sum; the genetic sequences all have the same length) using the new diagonal method, let $\mathbf{n}=(n, \ldots, n), I=1 / 2$ and $J=1-(1 / 2) \prod_{i=1}^{d}\left(1+x_{i}\right)$. Then CRIT(n), the solution set of $J=0$ and $n x_{i} \partial_{i} J=n x_{d} \partial_{d} J(i<d)$ comprises $\left(2^{1 / d} \omega_{i}-1, \ldots, 2^{1 / d} \omega_{i}-1\right)$, where the $\omega_{i}(i<d)$ are the dth roots of unity. By Theorem 3.1 . $\mathbf{c}:=\left(2^{1 / d}-1, \ldots, 2^{1 / d}-1\right)$ is the only contributing point. It is also a smooth point. By Proposition 3.4 . $\operatorname{det} \mathbf{H}=d 2^{(1-d) / d}$, and by Theorems 3.2 and 3.3 .

$$
F_{n \ldots n} \sim\left(2^{1 / d}-1\right)^{-d n} \frac{1}{\left(2^{1 / d}-1\right) 2^{\left(d^{2}-1\right) / 2 d} \sqrt{d(\pi n)^{d-1}}},
$$

in agreement with $\left(\overline{G H O W 90)}\right.$. (Please note the typo therein: the $2^{\left(k^{2}-1\right) / 2 k}$ in the formula on page 139 should be a $\left.2^{\left(1-k^{2}\right) / 2 k}\right)$.

A more important case for biological applications is to count alignments whose minimum block size is bounded below by a constant. A block of size $b$ in a $(d, \cdot)$-alignment is a $d \times b$ submatrix of the alignment 
with contiguous columns, none of which contain a zero. As shown in $(\mathbb{R T})$, the generating function for $(d, \cdot)$-alignments with blocks of size at least $b$ is

$$
\begin{aligned}
G\left(x_{1}, \ldots, x_{d}\right) & =\frac{1-t+t^{b}}{1+(1-p)\left(1-t+t^{b}\right)-t^{2}+t^{b+1}} \\
& =\frac{1-t+t^{b}}{1-t-(p-1-t)\left(1-t+t^{b}\right)}
\end{aligned}
$$

where $p=\prod_{i=1}^{d}\left(1+x_{i}\right)$ and $t=\prod_{i=1}^{d} x_{i}$. Note that when $b=1, G=F$, as expected.

To compute the asymptotics of $G_{n, \ldots, n}$ (which to our knowledge has only been done up till now for $d=2$ via the standard diagonal method $(\underline{R T})$ let $\mathbf{n}=(n, \ldots, n)$ and $J=1-t-(p-1-t)\left(1-t+t^{b}\right)$. Then CRIT(n) comprises all vectors $x \mathbf{1}$ such that $j(x):=J(x \mathbf{1})=0$ (as seen from the equations $\left.n x_{i} \partial_{i} J=n x_{d} \partial_{d} J\right)$. Let $\mathbf{c}:=c \mathbf{1}$ be the critical point in the positive orthant of $\mathbb{R}^{d}$ (so $c$ is the unique positive solution of $j(x)=0$ ) and note that $c<1$, because $j(x)<0$ for $x \geq 1$. By Theorem 3.1. $\mathbf{c} \in \operatorname{CONTRIB}(\mathbf{n})$ and all other points of $\operatorname{CONTRIB}(\mathbf{n})$ lie on the same torus as $\mathbf{c}$. Since

$$
G\left(x_{1}, \ldots, x_{d}\right)=\frac{1+\frac{t^{b}}{1-t}}{1-(p-1-t)\left(1+\frac{t^{b}}{1-t}\right)},
$$

on the open polydisk $\left\{\mathbf{x} \in \mathbb{C}^{d}: \forall i\left|x_{i}\right|<1\right\}$, since this polydisk contains the torus of $\mathbf{c}$, and since $(p-1-t)\left(1+\frac{t^{b}}{1-t}\right)$ is an aperiodic power series with nonnegative coefficients, we can apply Theorem 3.1 to conclude that $\mathbf{c}$ is the only contributing point. It is also a smooth point, as is seen most easily from the form $J=1-(p-1-t)\left(1+\frac{t^{b}}{1-t}\right)$. Thus by Theorem 3.2 .

$$
G_{n \ldots n} \sim c^{-d n} b_{0} n^{(1-d) / 2},
$$

where $b_{0}$ is the constant given in Theorem 3.3 Note that both $c$ and $b_{0}$ depend on $b$.

\section{References}

[BK86] Egbert Brieskorn and Horst Knörrer. Plane algebraic curves. Birkhäuser Verlag, Basel, 1986. Translated from the German by John Stillwell.

[BP] Yuliy Baryshnikov and Robin Pemantle. Convolutions of inverse linear functions via multivariate residues. preprint available at http://wWw. math. upenn.edu/ pemantle/ papers/Preprints/hyperplanes.pdf.

[FS] Phillipe Flajolet and Robert Sedgewick. Analytic combinatorics. In preparation, preprint available at http://algo.inria.fr/flajolet/Publications/ book061023.pdf

[Fur67] Harry Furstenberg. Algebraic functions over finite fields. J. Algebra, 7:271-277, 1967.

[GHOW90] J. R. Griggs, P. Hanlon, A. M. Odlyzko, and M. S. Waterman. On the number of alignments of $k$ sequences. Graphs Combin., 6(2):133-146, 1990. 
[HK71] M. L. J. Hautus and D. A. Klarner. The diagonal of a double power series. Duke Math. J., 38:229-235, 1971.

[Lip88] L. Lipshitz. The diagonal of a D-finite power series is D-finite. J. Algebra, 113(2):373-378, 1988.

[MZS04] Emanuele Munarini and Norma Zagaglia Salvi. Binary strings without zigzags. Sém. Lothar. Combin., 49:Art. B49h, 15 pp. (electronic), 2002/04.

[PW] Robin Pemantle and Mark C. Wilson. Twenty combinatorial examples of asymptotics derived from multivariate generating functions. To appear in SIAM Review, preprint available at http: / / arxiv.org/pdf/math.co/0512548.

[PW02] Robin Pemantle and Mark C. Wilson. Asymptotics of multivariate sequences. I. Smooth points of the singular variety. J. Combin. Theory Ser. A, 97(1):129-161, 2002.

[PW04] Robin Pemantle and Mark C. Wilson. Asymptotics of multivariate sequences. II. Multiple points of the singular variety. Combin. Probab. Comput., 13(4-5):735-761, 2004.

[RT] M. Régnier and F. Tahi. Generating functions in computational biology. Preprint available at http://algo.inria.fr/regnier/publis/ReTa04.ps.

[Sta99] Richard P. Stanley. Enumerative combinatorics. Vol. 2, volume 62 of Cambridge Studies in Advanced Mathematics. Cambridge University Press, Cambridge, 1999. With a foreword by Gian-Carlo Rota and appendix 1 by Sergey Fomin. 